\title{
Evaluación in vitro de la dureza superficial en amalgamas y coronas de acero cromado
}

Natalia Gutiérrez Marín ${ }^{1}$;

Andrea López Soto ${ }^{2}$

\section{Resumen}

Introducción: la elección del material restaurador es uno de los puntos críticos cuando se va a restaurar un diente temporal. Tradicionalmente, la corona de acero cromado ha sido empleada en dientes con extensa destrucción coronal o posterior a un tratamiento pulpar; no obstante, la amalgama también ha sido muy utilizada. Existen estudios que miden el porcentaje de éxito a nivel clínico de dichos materiales, sin embargo, no hay investigaciones que midan y comparen las propiedades físicas. Objetivo: evaluar la dureza superficial en amalgamas y coronas de acero cromado. Materiales y método: se confeccionaron 15 cuerpos de prueba de amalgama SDI tipo GS80, y 15 cuerpos de prueba con un trozo de corona de acero cromado marca 3M. Los cuerpos de prueba se elaboraron en dos tiempos diferentes, unos fueron alma- cenados por 4 años y los otros por 3 días al 100\% de humedad relativa. A cada cuerpo de prueba se le hicieron 2 indentaciones con el microdurómetro Micromet 2001 para determinar la dureza superficial. Los resultados obtenidos fueron evaluados por el test ANOVA $(p \leq 0,05)$ Resultados: se comprobó que la media de la dureza superficial de la corona de acero cromado es significativamente mayor que la media de la amalgama ( $p=0,000)$. La dureza superficial de la amalgama aumenta de forma significativa con el tiempo de almacenamiento $(p=0,000)$. Conclusiones: el material que presentó mayor dureza superficial fue la corona de acero cromado. Los valores de microdureza de la amalgama son tiempo-dependientes, no así los valores registrados con las coronas de acero cromado.

Palabras claves: Dureza superficial, amalgama, corona de acero cromado.

\section{Artigo Original}

\section{Avaliação in vitro da dureza superficial em amálgamas e coroas de aço}

\section{Resumo}

Introdução: a seleção do material restaurador é um ponto crítico no momento da restauração de dentes decíduos.Tradicionalmente, a coroa de aço tem sido utilizada em dentes com muita destruição coronária ou após tratamento endodôntico, porém o amálgama pode também ser utilizado nesses casos. Existem estudos que determinam a porcentagem de sucesso a nível clínico desses materiais, no entanto, poucas pesquisas medem e comparam as propriedades físicas. Objetivo: avaliar a dureza 
superficial em amálgamas dentais e coroas de aço. Materiais e método: foram elaborados 15 corpos de prova de amálgama SDI tipo GS80, e 15 corpos de prova com um fragmento de coroa de aço marca 3M. A fabricação dos corpos de prova ocorreu em dois tempos distintos, sendo alguns armazenados por 4 anos e outros por 3 dias a $100 \%$ de umidade relativa. A dureza superficial foi determinada a partir de duas endentações feitas com o microdurômetro Micromet 2001 em cada corpo de prova. Os resultados foram avaliados com o teste de ANOVA $(\mathrm{p} \leq 0,05)$. Resultados: a média da du- reza superficial da coroa de aço foi significativamente maior que a média da dureza do amálgama $(p=0,000)$. A dureza superficial do amálgama aumenta significativamente conforme o tempo de armazenamento $(p=0,000)$. Conclusões: a coroa de aço foi o material que apresentou maior dureza superficial. Os valores da microdureza do amálgama são tempo-dependentes, diferentemente dos valores da coroa de aço.

Palavras-chave: Dureza superficial, amálgama, coroa de aço.

\section{Original article}

\section{In vitro evaluation of micro-hardness of amalgam and stainless-steel crowns}

\begin{abstract}
Introduction: the choice of the restorative material is critical for the restoration of primary teeth. Traditionally, stainless-steel crowns have been used in teeth with extensive crown destruction or after pulp treatment; however amalgam can be used too. There are studies that have measured the clinical success of these materials; however, there are no reports that have measured and compared the physical properties between them. Aim: evaluate the micro-hardness of dental amalgam and stainless steel crown. Method and materials: test objects of SDI amalgam type GS80 (15) and 3M stainless-steel crown (15) were made in two different times, some were stored for 4 years and the others for 3 days at $100 \%$ relative humidity. Two indentations were made to each test object with the Micromet 2001 microdurometer to determine the micro-hardness. The results obtained were evaluated by the ANOVA test ( $p \leq 0.05)$. Results: the average of the microhardness stainless steel crown was significantly

higher than the average of the amalgam $(\mathrm{p}=0.000)$. The micro-hardness of the amalgam increases significantly with the storage time $(\mathrm{p}=0.000)$. Conclusions: the stainless-steel crown was the material with the highest micro-hardness. The micro-hardness values of the amalgam are time-dependent.

Keywords: Micro-hardness, dental amalgam, stainless steel crown.

\section{Introducción}

La preservación de los dientes primarios es esencial para la función masticatoria, la fonética, la estética, la conservación del espacio en el arco y guía de la dentición permanente. ${ }^{1,2}$ La restauración de dientes muy destruidos por caries y con poca estructura dental remanente, así como aquellas con tratamiento pulpar, son una gran preocupación en la odontología pediátrica. Numerosas investigaciones han demostrado que el éxito de una pulpotomía está
\end{abstract}


íntimamente relacionado con la restauración final del diente. . $^{3-6}$

La selección del material restaurador depende de varios factores: evaluación del riesgo de caries, el estado de desarrollo de la dentición, la cooperación del paciente durante el tratamiento y las propiedades del material. ${ }^{7}$ En odontopediatría, el material considerado como patrón oro en las restauraciones de dientes primarios con terapia pulpar o gran destrucción coronal es la corona de acero cromado (CAC) ya que minimiza las posibilidades de fractura del diente. ${ }^{3,4,8-10}$

Un estudio realizado para determinar preferencia de materiales para restauración en dentición primario encontró que en lesiones clase II, la mitad de los profesionales emplean como primera opción las amalgamas y en segundo lugar las resinas compuestas; mientras que para lesiones de ambas superficies proximales con posible compromiso pulpar, la mayoría de los profesionales utilizan las CAC seguido por las amalgamas. ${ }^{11}$ Esta preferencia por CAC puede estar relacionada con su superioridad en la reducción de fracaso por dolor, infección, patologías periradiculares o desajuste de la restauración a largo plazo en comparación con restauraciones de amalgama. ${ }^{12}$ Por otra parte, una investigación señala que la amalgama sigue siendo ampliamente utilizada por los odontólogos para restaurar dientes primarios. ${ }^{13}$

A parte de las preferencias de los materiales por parte de los odontólogos y de las investigaciones en términos de fracaso clínico, en la literatura no se encuentran estudios reportados que midan y comparen las propiedades físicas de amalgamas y CAC para justificar la superioridad de una sobre la otra. Una de las características que se utiliza para evaluar y comparar la superioridad entre materiales es la dureza superficial, la cual representa la resistencia superficial de un cuerpo a la indentación o rayado, y es el resultado de la confluencia de varias propiedades: ductilidad, maleabilidad, resistencia, límite proporcional, resistencia a la abrasión y al corte. ${ }^{14}$ Por lo tanto, el objetivo de este estudio es evaluar la dureza superficial de la amalgama y la corona de acero cromado para determinar si existen diferencias entre los materiales.

\section{Materiales y metodo}

Se seleccionó la CAC preformada de la marca $3 \mathrm{M}$ y la amalgama marca SDI tipo GS80.

\section{Confección de cuerpos de prueba}

Para la obtención de cuerpos de prueba fueron utilizadas matrices cilíndricas de acrílico que contenían un orificio central de $3 \mathrm{~mm}$ de diámetro por $2 \mathrm{~mm}$ de profundidad. Fueron confeccionados 15 cuerpos de prueba para cada material. Para confeccionar los cuerpos de amalgama, se utilizó amalgama marca SDI tipo GS80; la amalgama se mezcló en un equipo Gnatus Amalga Mix II por 15 segundos, se empacó el material en las matrices cilíndricas de acrílico, se condensó con un empacador y se bruñó con un instrumento de bolita (ambos marca Hu-Fridey).Para los cuerpos de prueba de la CAC, se cortó un trozo pequeño de la superficie lingual de una CAC de una molar, el trozo se asentó sobre cemento de ionómero de vidrio Relyx Luting 2 de la marca 3M ESPE en las matrices acrílicas (Figura 1). Una vez elaborados todos los cuerpos de prueba, se almacenaron a temperatura ambiente en un recipiente plástico con agua al $100 \%$ de humedad relativa. Tanto los cuerpos de amalgama como 
los de la CAC, se confeccionaron en dos tiempos diferentes, el primer grupo fue almacenado por 4 años (2012) y el segundo grupo por tres días (2016).

Se decidió realizar pruebas a los materiales en un intervalo de 4 años por dos razones: primero, debido a las modificaciones reportadas en la dureza superficial de la amalgama con el tiempo; ${ }^{15}$ y segundo, ya que existen investigaciones que han demostrado que la mayor prevalencia de caries en dentición primario se presenta entre los 6 y 7 años; ${ }^{16,17}$ así que, si una molar se restaura a esa edad, la permanencia en boca hasta la exfoliación sería alrededor de los 4 años si se aplican las tablas internaciones de exfoliación y erupción dental. ${ }^{1,2}$ Por lo tanto, se desea conocer el comportamiento de los materiales en ese lapso del tiempo.

Previo a realizar las pruebas de dureza se realizó una calibración intraobservador para validar las medidas del área de la indentación. No se encontró diferencia estadísticamente significativa en el área medida $(\mathrm{p}=0,844)$.

Todos los cuerpos de prueba se sometieron al test de microdureza en un microdurómetro Micromet 2001 marca Buehler propiedad de la Escuela de Física de la Universidad de Costa Rica. El aparato consta de una punta piramidal de diamante con un ángulo de 136 grados al que se le estipula una
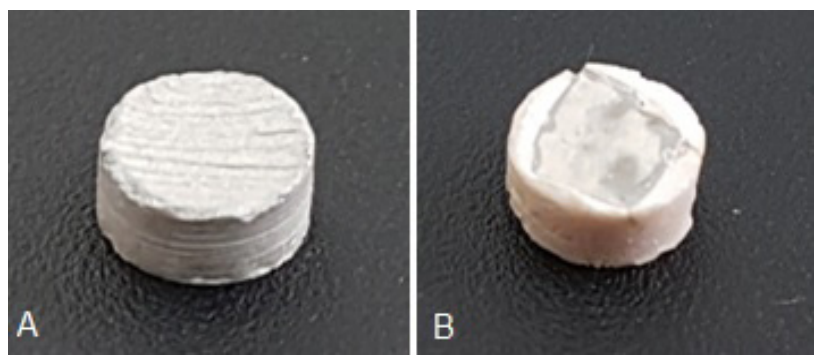

Figura 1. Cuerpos de prueba. A. Amalgama B. Corona de acero cromado
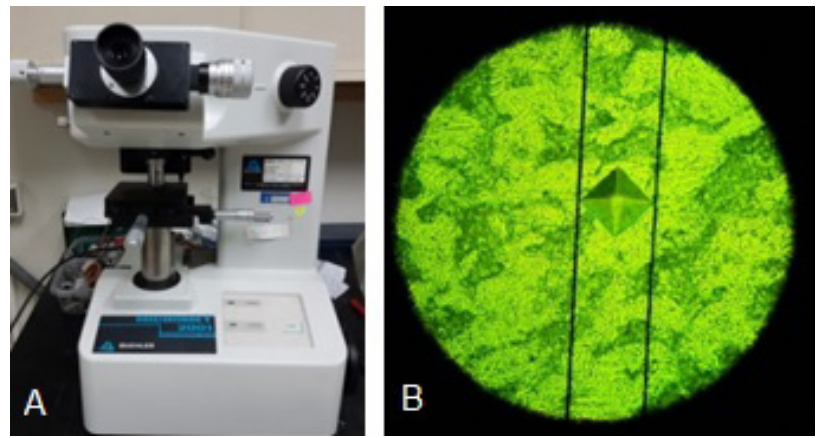

Figura 2. A. Micromet 2001. B. Indentación vista en el microscopio.

carga de 500 gr por 20 segundos. Se realizaron dos indentaciones por separado en la parte superior de cada cuerpo de prueba; se obtuvo un promedio de las impresiones de cada cuerpo de prueba. Con los resultados obtenidos de las medidas de dureza se calculó la dureza Vickers de cada indentación. (Figura 2).

\section{Metodología estadística}

Los datos fueron analizados empleando el programa SPSS Statistical Package for the Social Sciences (Versión 19.0 SPSS Inc., Chicago, IL, EUA). Para determinar las diferencias entre las medias de dureza Vickers de los dos materiales en los diferentes tiempos de fabricación fue utilizado el método de análisis de varianza, con un nivel de significancia de $p<0.05$. Esto después de realizar la prueba de homogeneidad de varianza y no encontrar diferencia estadísticamente significativa $(p=0,839)$.

\section{Resultados}

En la Tabla 1, se observan los promedios, mediana y rango de la dureza superficial de la amalgama y la CAC para los años estudiados. 


\begin{tabular}{|c|c|c|c|c|}
\hline Material/ Año & $\mathbf{N}$ & $\begin{array}{c}\text { Promedio } \\
\text { (desviación } \\
\text { estandar) }\end{array}$ & Mediana & $\begin{array}{c}\text { Rango dureza } \\
\text { Vickers }\end{array}$ \\
\hline Amalgama/ 2012 & 15 & $129.90(2.68)$ & 130.38 & $122.58-132.90$ \\
\hline Amalgama/2016 & 15 & $119.57(2.29)$ & 119.23 & $116.25-123.39$ \\
\hline CAC/ 2012 & 15 & $161.48(2.02)$ & 161.58 & $157.79-165.21$ \\
\hline CAC/2016 & 15 & $162.06(2.27)$ & 162.28 & $157.81-166.65$ \\
\hline
\end{tabular}

Tabla 1. Promedio, mediana y rango de dureza Vickers de la amalgama y la CAC.

Se encontró diferencia estadísticamente significativa al comparar la media de la dureza superficial entre los materiales ( $\mathrm{p}=0.000$ ), siendo mayor la dureza de la CAC. Al comparar por años también se encuentra una diferencia significativa $(p=0.000)$. En la interacción entre año y material, la diferencia significativa se encuentra en la amalgama ( $\mathrm{p}=0.000)$ y no así la CAC ( $\mathrm{p}=0.503)$, por lo tanto, la amalgama que tenía más tiempo de estar almace-

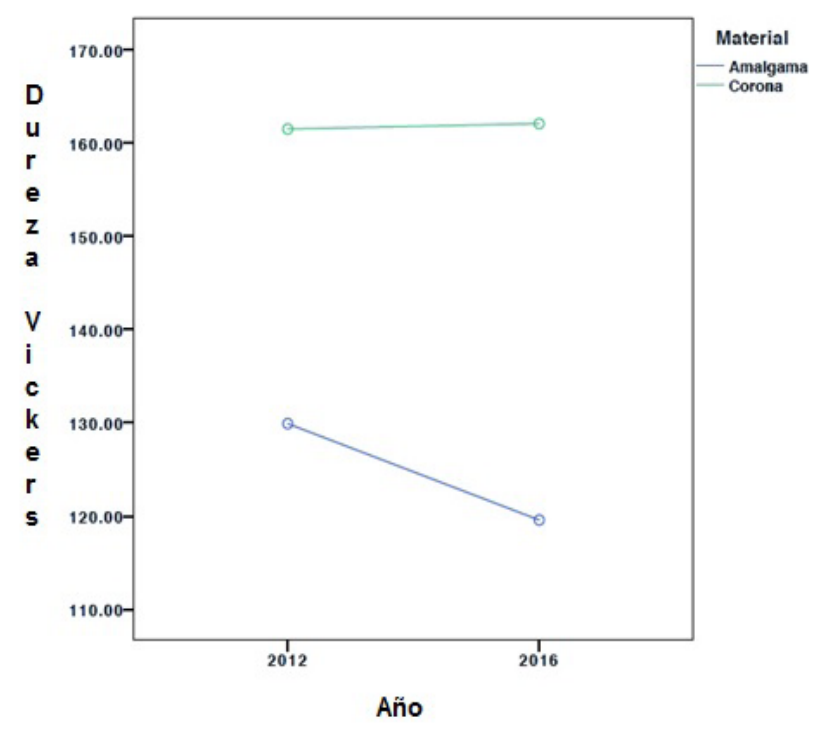

Gráfico 1. Media de la dureza Vickers de CAC y amalgamas en los añoos 2012-2016. nada presentó una dureza mayor con respecto a la que tenia 3 días de fabricada.(Gráfico 1).

\section{Discusión}

Son pocos los estudios que evalúan las propiedades físicas de las amalgamas y CAC, algunas investigaciones se enfocan en la resistencia al desalojo y dureza superficial en las amalgamas; ${ }^{15,18,19}$ y otros en la resistencia de las CAC al ser sometidas a fuerzas de compresión, torsión y corte. ${ }^{20}$ Pero ninguno ha determinado la dureza superficial de la CAC y comparado los resultados entre los dos materiales.

Nuestros resultados demuestran que el material con mayor dureza superficial fue la corona de acero cromado. Dicho resultado puede tener concordancia con varios estudios que han comparado a nivel clínico la durabilidad y longevidad de las coronas de acero cromado y las restauraciones de amalgamas clase II, demostrando la superioridad de las coronas de acero cromado en ambos parámetros. ${ }^{7,11,21-23}$ Por ejemplo, de acuerdo con una revisión de literatura, el porcentaje de fallo a cinco 
años de una CAC fue de un $7 \%$ frente a un $26 \%$ en restauraciones de amalgama clase II. ${ }^{11}$

Respecto a dientes primarios en los cuales se realizaron tratamientos pulpares y luego se restauraron con diversos materiales, los porcentajes de éxito son variados. Existen investigaciones donde no se hallaron diferencias significativas en el éxito de dientes con pulpotomías restaurados con amalgama o CAC. 5,6 Sin embargo, un estudio clínico longitudinal durante ocho años donde se estudiaron restauraciones de múltiples superficies, mostró un éxito del 89\% en piezas restauradas con CAC frente a un $80 \%$ de éxito en aquellas donde se empleó la amalgama. ${ }^{23}$

Adicionalmente, un estudio de Sonmez y Duruturk donde investigaban el éxito de dientes con pulpotomía y posteriormente CAC o amalgamas, hallaron mayor éxito cuando se había colocado una corona de acero cromado $97,6 \%$ que una amalgama $85,7 \% .{ }^{4}$ En los estudios citados, los dientes que menos fallas presentaron fueron aquellas restauradas con CAC, lo cual también está respaldado por una revisión sistemática de literatura realizada por Seale donde se recomienda el uso de CAC en dientes con lesiones por caries muy extensas debido a su comprobado éxito. 25

El mayor porcentaje de éxito de los dientes con tratamiento pulpar y CAC que ha sido descrito, podría tener relación con la mayor dureza superficial de las CAC, ya que son utilizadas en dientes con lesiones cariosas grandes que deben ser restauradas para soportar altas cargas de masticación. Además, un estudio concluye que las CAC evitan la fractura del diente durante los movimientos de masticación porque adsorben la mayoría de las fuerzas y permiten que fuerzas mínimas alcancen la dentina. ${ }^{21}$
En relación a la amalgama, los resultados de dureza superficial obtenidos son similares a los reportados en la literatura. ${ }^{15,19,25}$ Además, el presente estudio indica un aumento en la dureza superficial de la amalgama con el tiempo, lo cual coincide con los resultados de Ribeiro et al. y de Perdigoni et al.; quienes hallaron mayor dureza superficial en los especímenes con mayor tiempo de fabricación.

Una de las limitaciones del estudio fue que las pruebas se realizaron in vitro y no reflejan las condiciones reales de la cavidad oral; sin embargo, se da a conocer información que no se tenía previamente respecto a la comparación de propiedades físicas de algunos materiales altamente utilizados en odontopediatría, a la vez que se da sustento científico al uso de la corona de acero cromado.

Por otra parte, se pueden diseñar estudios que midan además la dureza superficial de resinas, coronas con frente estético y de zirconia. Las coronas de zirconia han tomado relevancia en los últimos años por ser una alternativa estética y los reportes de investigaciones se han centrado en la resistencia a la fractura, dejando de lado la medición de la dureza superficial. ${ }^{26,27}$

\section{Conclusiones}

El material que presentó mayor dureza superficial fue la corona de acero cromado. Los valores de dureza de la amalgama son tiempo dependientes, no así los valores registrados con las coronas de acero cromado.

La mayor dureza superficial de la corona de acero cromado, es una propiedad física que se puede asociar al éxito clínico de dicho material en dientes con lesiones cariosas extensas o con tratamiento pulpar. 


\section{Referencias bibliográficas}

1. Pinkhan JR, Casamassimo PS, Fields HW, McTigue, DLNowak A. Odontología Pediátrica. $3^{\circ}$ ed. México DF: McGraw-Hill Interamericana; 2001.

2. McDonald R, Avery D. Odontología pediátrica y del adolescente. $6^{\circ}$ ed. Madrid: Harcourt Brace; 1998.

3. Hutcheson C, Seale S, McWhorter A, Kerins C, Wright J. Multi-surface Composite vs Stainless Steel Crown Restorations After Mineral Trioxide Aggregate Pulpotomy: A Randomized Controlled Trial. Pediatr Dent. 2012; 34: 460-7.

4. Somnez D, Duruturk L. Success rate of calcium hydroxide pulpotomy in primary molars restored with amalgam and stainless steel crowns. British Dental J. 2010; 208: E18 1-5.

5. Holan G, Fuks A, Keltz N. Success rate of formocresol pulpotomy in primary molars restored with stainless steel crown vs amalgam. Pediatr Dent. 2001; 24: 212-6.

6. Haghgoo R, Abbasi F. Clinical and Radiographic Evaluation of Pulpotomized Primary Molars Restored with Stainless Steel Crown and Amalgam. Shiraz Univ Dent J. 2011; 12: 221-6.

7. Dhar V, Coll JA, Ginsber E, Ball BM, Chhibber S, Johnson M. Evidence-based Update of Pediatric Dental Restorative Procedures: Dental Materials. J Clin Pediatr Dent. 2015; 39: 303-10.

8. Croll T, Killian C. Zinc oxide-eugenol pulpotomy and stainless steel crown restoration of a primary molar. Quintessence Int. 1992; 23: 383-8.

9. Ross R. Preformed metal crowns for primary and permanent molar teeth: review of literature. Pediatr Dent. $2002 ; 24: 489-500$.

10. American Academy of Pediatric Dentistry. Guideline on Pediatric Restorative Dentistry. Reference Manual. Pediatr Dent. 2014; 37: 226-34.

11. Al-Dlaigan Y. Pediatric dentist's choices of restorative materials for primary molars. Pakistan oral and dental J. 2015; 35: 83-7.

12. Innes NPT, Ricketts D, Chong LY, Keightley AJ, Lamont T, Santamaria RM. Preformed crowns for decayed primary molar teeth.๑Cochrane Database of Systematic Reviews 2015, Issue 12. Art. No.: CD005512. DOI: 10.1002/14651858.CD005512.pub3.

13. Blumer S, Peretz B, Ratson T. The Use of Restorative Materials in Primary Molars among Pediatric Dentistry in Israel. J Clin Pediatr Dent. 2017; 41: 424-8.

14. Guzmán H. Polímeros, Resinas Compuestas. Biomateriales Odontológicos de uso clínico.4 ed. Bogotá: Ecoe; 2006. P. 175208.

15. Ribeiro S, Nascimento T, Centola A, Teixeira L, Maia S. Effect of Polishing Burns and Stones on the Micro-Hardness of Dental Amalgam. Braz Den J. 1991; 2: 135-43.

16. Reddy KS, Reddy S, Ravindhar P, Balaji K, Reddy H, Reddy A. Prevalence of dental caries among 6-12 years school children of Mahbubnagar District, Telangana State, India: A cross-sectional study. Indian J Dent Sci. 2017; 9: 1-7.

17. Bobu L, Barlean L, Murariu A, Barlean M. Caries-risk Evaluation of Schoolchildren in Iasi, Romania. Romanian J of Oral Rehab. 2017; 9: 101-6.

18. Barceló F, Velásquez N, Guerrero J. Resistencia al desalojo por empuje de materiales restaurativos directos. Rev Odont Mex. 2005; 9: 178-84.

19. Perdigoni M, Centola A, Froner I, Turbino M, Ribeiro S. Effect of the Polishing Technique at Low or High Speed on the Micro-hardness of Dental Amalgam. Braz Den J. 1991; 2: 51-7.

20. Prabhakar A, YavagalCh, Chakraborty A, Sugandhan S. Finite Element Stress Analysis of Stainless Steel Crowns. J Indian Soc Pedod Prev Dent. 2015; 33: 183-91.

21. Attari N, Roberts JF. Restoration of primary teeth with crowns: a systematic review of the literature. Eur Arch Paediatr Dent. 2006; 7: 58-62. 
Gutiérrez Marín N, López Soto A.

22. Threfall AG, Pilkington L, Milson KM, Blinkhorn AS, Tickle M. General dental practitioners' views on the use of stainless steel crowns to restore primary molars. Br Dent J, 2005; 199: 453-455.

23. Einwag J, Dünninger P. Stainless steel crown versus multisurface amalgam restorations: An 8-year longitudinal clinical study. Quintessence Intl. 1996; 27: 321-23.

24. Seale S, Ross R. The Use of Stainless Steel Crowns: a Systematic Literature Review.Pediatr Dent 2015: 37 : 147-62.

25. Nakai H, Ishizaki N, Nihei I. The microestructure and hardness of dental amalgam. J Osaka D Univ. 1970; 4: 131-149.

26. Beattie S, Taskonak B, Jones J, Chin J, Sanders B, Tomlin A, Weddell J. Fracture resistance of 3 types of primary esthetic stainless steel crowns. J Can Dent Assoc 2011; 77: b90.

27. Townsend JA; Knoell P, Yu Q, Zhang JF, Wang Y, Zhu H, Beattie S, Xu X. In vitro fracture resistance of three commercially available zirconia crowns for primary molars. Pediatr Dent. 2014; 38: 125-9.

Recibido: 05/09/2017

Aceptado: 12/01/2018

Correspondencia: Natalia Gutiérrez Marín. gutierreznatalia@hotmail.com. 